\title{
DIVERSIDAD DE CERVIDAE (MAMMALIA, ARTIODACTYLA) EN EL PLEISTOCENO DE LA PROVINCIA DE CORRIENTES, ARGENTINA
}

\author{
MARÍA ALEJANDRA ALCARAZ \\ Centro de Ecología Aplicada del Litoral y Consejo Nacional de Investigaciones Científicas y Tecnológicas, CONICET, \\ Ruta 5, km 2,5, CC 128, Corrientes, Argentina. alejandralcaraz@gmail.com
}

ANALÍA FRANCIA

División Paleontología de Vertebrados, Facultad de Ciencias Naturales y Museo de La Plata, Universidad Nacional de La Plata, Paseo del Bosque s/n, 1900, La Plata, Argentina. analiafrancia@yahoo.com.ar

\begin{abstract}
DIVERSITY OF CERVIDAE (MAMMALIA, ARTIODACTYLA) IN THE PLEISTOCENE OF CORRIENTES PROVINCE, ARGENTINA. The study presents an analysis of the peculiar diversity of cervids recorded in two localities at the Corrientes Province (Argentina), Lavalle and Bella Vista. The sediments belong to two successive formations of the late Pleistocene (Lujanian floor/age): Toropí Formation ( $c a .52 .000$ years BP) and Yupoí Formation ( $c a .36 .000$ years BP). The remains are in general antlers, except for a specimen that also preserves partial skull and postcranium. Morenelaphus lujanensis (Ameghino), Antifer ultra (Ameghino), A. ensenadensis (Ameghino), and Hippocamelus sulcatus (Ameghino) are recorded in Toropí Formation, whereas the Yupoí Formation yielded cf. Mazama sp. and Morenelaphus sp. The following issues are discussed: the great diversity of species that include two large forms, A. ultra and A. ensenadensis, and one median to large-size form, M. lujanensis; the record for cf. Mazama in the Pleistocene of Argentina, previously restricted to the Holocene-Present; and the presence of $A$. ensenadensis in the late Pleistocene outside the Pampean region, expanding its geographic distribution towards northeast Argentina and its biochron beyond the early-middle Pleistocene. Such diversity is remarkable considering other cervid faunas recorded in the late Pleistocene of geographically close areas, like Entre Ríos, Argentina (El Palmar and Tezanos Pinto formations), Uruguay (Sopas Formation), and Southern Brazil (Touro Passo Formation). The greatest specific richness is found in Toropí Formation, which is comparable to that of the late Pleistocene of Sopas Formation, although the age of sediments and the species yielded are not the same.
\end{abstract}

Key words: Corrientes Province, Pleistocene, Cervidae, diversity, paleobiogeography.

RESUMO - Uma análise sobre a particular diversidade de cervídeos registrados em duas localidades da Província de Corrientes, Argentina, Lavalle e Bella Vista é aqui apresentada. Os sedimentos pertencem a duas formações sucessivas do Pleistoceno tardio: Formação Toropí (ca. 52.000 anos AP) e Formação Yupoí (ca. 36.000 anos AP). Os restos são em geral hastes, exceto os de um exemplar que também conservou parte do crânio e pós-crânio. Para a Formação Toropí são registradas Morenelaphus lujanensis (Ameghino), Antifer ultra (Ameghino), A. ensenadensis (Ameghino) e Hippocamelus sulcatus (Ameghino); e para a Formação Yupoí, cf. Mazama sp. e Morenelaphus sp. É discutida a grande diversidade de espécies que incluem duas formas de grande tamanho, como Antifer ultra e A. ensenadensis e outra de médio a grande porte, M. lujanensis; o registro de cf. Mazama no Pleistoceno da Argentina, restringido previamente ao Holoceno-Atual; e o achado de A. ensenadensis em sedimentos do Pleistoceno tardio fora da região Pampeana, permitindo ampliar sua distribuição geográfica para o nordeste da Argentina e seu intervalo temporal além do Pleistoceno inicial-médio. Tal diversidade é chamativa considerando outras faunas de cervídeos registradas no Pleistoceno tardio em áreas geograficamente próximas, como a Província de Entre Rios, Argentina (formações El Palmar e Tezanos Pinto); Uruguai (Formação Sopas) e sul do Brasil (Formação Touro Passo). A maior riqueza específica se registra na Formação Toropí, que é comparável com a ocorrida durante o Pleistoceno tardio na Formação Sopas, ainda que as idades dos sedimentos portadores, assim como as espécies presentes em ambas formações, não sejam as mesmas.

Palavras-chave: Província de Corrientes, Pleistoceno, Cervidae, diversidade, paleobiogeografía.

\section{INTRODUCCIÓN}

El Gran Intercambio Biótico Americano, un evento biológico gradual entre América del Sur y América del Norte (Stehli \& Webb, 1985), permitió el intercambio faunístico bidireccional de varios grupos de mamíferos, y entre ellos, el ingreso de los Cervidae al continente sudamericano. Esta familia constituyó, junto a otros grupos de mamíferos, el tercer estrato de inmigrantes (Marshall et al., 1984), que se distribuyó ampliamente por Sudamérica durante el Pleistoceno. El registro paleontológico de los cérvidos ha sido documentado en sedimentos cuaternarios del Paraguay (Carlini \& Tonni, 
2000), del Uruguay (Kraglievich, 1932; Ubilla et al., 2009; Corona \& Ubilla, 2010), de Brasil (Bombín, 1976; Paula Couto, 1979; Souza Cunha \& Magalhães, 1981; Oliveira, 1992; Scherer et al., 2007; Ribeiro \& Scherer, 2009; Guerin \& Faure, 2009); de Chile (Casamiquela, 1976, 1984, 1999; Labarca \& Alcaraz, 2011), de Bolivia (Ameghino, 1902; Hoffstetter, 1963), de Ecuador (Wolf, 1875; Hoffstetter, 1952; Tomiati \& Abbazzi, 2002), de Perú (Churcher, 1962; Hoffstetter, 1986), de Colombia (Marshall et al., 1984) y de Venezuela (Marshall et al., 1984; Aguilera \& Orangel, 2004). Actualmente, las especies de cérvidos sudamericanos se distribuyen desde los $52^{\circ} \mathrm{S}$ hasta $\operatorname{los} 10^{\circ} \mathrm{N}$ de latitud, y entre los $80^{\circ}$ y $38^{\circ}$ longitud oeste, con una distribución altitudinal hasta los $5.200 \mathrm{msnm}$ (Emmons, 1999; Canevari \& Vaccaro, 2007; Herreros de Lartundo \& Sielfeld Kowald, 2011). En la Argentina, el conocimiento acerca de la diversidad de ciervos extinguidos y actuales en sitios arqueológicos y paleontológicos se encontraba acotado a la Provincia de Buenos Aires (Menegaz, 2000). Sin embargo, recientemente uno de los autores (MAA) extendió su estudio al resto de las áreas extraandinas de la Argentina (Alcaraz, 2010). Para la Provincia de Corrientes, Álvarez (1974), Zurita \& Lutz (2002), Alcaraz \& Carlini (2003), Alcaraz \& Zurita (2004), Alcaraz \& Francia (2010), describieron parcialmente los restos de cérvidos exhumados en sedimentos correspondientes al Pleistoceno tardío. A partir de nuevas colecciones y de la revisión de las determinaciones previas, se actualiza la información conocida hasta el momento acerca de la diversidad de ciervos presentes durante el Pleistoceno tardío en la Provincia de Corrientes y se la analiza para un área geográfica y un lapso de tiempo muy acotados. Además, se compara esa diversidad con aquellas descriptas para sedimentos de la Provincia de Entre Ríos, Argentina (formaciones Tezanos Pinto y El Palmar, Ferrero, 2009; Ferrero et al., 2007), sur de Brasil (Formación Touro Passo, Bombin, 1976; Kerber \& Oliveira, 2008; Oliveira \& Kerber, 2009; Ribeiro \& Scherer, 2009) y norte de Uruguay (Formación Sopas, Ubilla 2004; Ubilla et al., 2009; Corona \& Ubilla, 2010) y se proponen inferencias sistemáticas, paleobiogeográficas, paleoecológicas y bioestratigráficas vinculadas.

\section{MATERIAL Y MÉTODOS}

Se estudiaron los restos de ciervos pleistocenos procedentes de la Provincia de Corrientes depositados en la colección de la Facultad de Ciencias Exactas y Naturales y Agrimensura de la Universidad Nacional del Nordeste (Corrientes, Argentina). Para el análisis anatómico de los mismos, se realizaron comparaciones morfológicas y morfométricas principalmente a partir de las características de las astas. Las medidas tomadas a cada ejemplar, así como los atributos morfológicos considerados en las determinaciones taxonómicas fueron extraídos de Alcaraz (2010). Para las comparaciones referentes a diversidad de ciervos en áreas geográficas cercanas se utilizaron los estudios faunísticos publicados previamente (e.g. Ferrero, 2009; Ferrero et al., 2007; Ubilla, 2004; Ubilla et al., 2009; Corona \& Ubilla, 2010; Bombin, 1976; Kerber \& Oliveira, 2008; Oliveira \& Kerber, 2009; Ribeiro \& Scherer, 2009). Este trabajo se basó en el estudio de las astas, en tanto son consideradas el elemento anatómico diagnóstico principal para las determinaciones sistemáticas de los ciervos extintos (Ameghino, 1889; Kraglievich, 1932; Bagnalasta, 1980). El ejemplar de Hippocamelus sulcatus (Ameghino, 1888) (CTES-PZ 7494) fue el único que además conservó parte del cráneo y elementos del postcráneo. Sin embargo esos materiales no se analizaron en detalle aquí, ya que forman parte de un estudio más extenso y minucioso de revisión sistemática de esta especie extinta, que se encuentra en preparación, dada la importancia del ejemplar.

Las astas fueron descritas de acuerdo a su posición en vida distinguiéndose en ellas un borde anterior (siempre cóncavo), un borde posterior (plano o algo convexo), una cara externa (convexa) y una cara interna (cóncava). Para una mejor comprensión, a continuación se detalla la nomenclatura morfológica utilizada en las descripciones siguiendo el criterio de Alcaraz (2010).

Base: es la superficie sobre la que las astas se unen a los ceratóforos (prolongaciones óseas de los huesos frontales) o a partir de la que se desprenden, cuando se producen los volteos anuales de las mismas.

Roseta: conjunto de pequeñas excrecencias o tubérculos óseos, que se disponen en círculo alrededor de la base de las astas.

Pedúnculo: porción proximal del asta por encima de la roseta, a partir del cual se proyectan las ramas anterior y la posterior del asta.

Rama anterior o garceta: es la primera rama del asta. Se ubica sobre el borde anterior de la misma y se dispone a distancia variable de la base dependiendo del género o especie de la que se trate.

Rama posterior o vara: es la rama principal del asta, en los ciervos que presentan apéndices multirramosos. Sobre ella se disponen el resto de las ramificaciones, cuyo número también es variable.

Crestas y surcos longitudinales: son estrías marcadas que recorren el asta en toda su extensión, desde la base siguiendo por la rama principal hasta su respectiva ramificación.

Abreviaturas. CTES-PZ, Colección de la Facultad de Ciencias Exactas y Naturales y Agrimensura de la Universidad Nacional del Nordeste, Corrientes, Argentina; MUFyCA, Museo Florentino y Carlos Ameghino de Rosario, Argentina; EIO, Estadio Isotópico del Oxígeno; OSL, Optically Stimulated Luminescence (Luminiscencia Ópticamente Estimulada); TL, termoluminiscencia.

\section{MARCO GEOGRÁFICO, GEOLÓGICO Y ESTRATIGRÁFICO}

La Provincia de Corrientes abarca una superficie aproximada de $88.000 \mathrm{~km}^{2}$ y se localiza en el noreste de la República Argentina, entre los $27^{\circ}$ y $30^{\circ}$ latitud sur y entre los $28^{\circ}$ y $30^{\circ}$ longitud oeste, constituyendo junto a las provincias de Misiones y Entre Ríos la región Mesopotámica (Figura 1). Los yacimientos cuaternarios que allí afloran, se extienden a lo largo de las costas del Río Paraná y se encuentran representados por dos formaciones sucesivas (Herbst \& Álvarez, 1977; Herbst \& Santa Cruz, 1999) del Pleistoceno tardío (ca. 126.000-11.700 años AP, sensu International Stratigraphic Chart, 2012): 
Formación Toropí (ca. 52.000 años AP) y Formación Yupoí (ca. 36.000 años AP) cuya antigüedad fue estimada a partir de análisis OSL (Tonni et al., 2005; Francia et al., 2012b). Siguiendo a Herbst \& Álvarez (1977) y Herbst \& Santa Cruz (1999), la Formación Toropí está constituida por arenas arcillosas, limos arenosos y arcillas arenosas. En todos los casos en los cuales se hace visible, se apoya sobre la Formación Ituzaingó (Plioceno), y es subyacente a la Formación Yupoí, conformada por arenas limosas, limos arenosos y arenas arcillosas, en general sin una estratificación visible, formando por acción de la erosión los llamados “tubos de órgano". Separa ambas formaciones una discordancia erosiva que no siempre es visible.

Por su parte, más recientemente, Iriondo $(1996,2010)$ a base de las similitudes mineralógicas de ambas formaciones y del carácter intraformacional de la discordancia erosiva, reúne en una sola formación que denominó Formación Toropí/ Yupoí, al conjunto sedimentario que Herbst \& Álvarez (1977) y Herbst \& Santa Cruz (1999) consideran como unidades litoestratigráficas independientes.

\section{SISTEMÁTICA PALEONTOLÓGICA}

Orden ARTIODACTYLA Owen, 1848

Suborden RUMINANTIA Scopoli, 1777

Infraorden PECORA Linnaeus, 1758

Familia CERVIDAE Gray, 1821

Tribu ODOCOILEINI Webb, 1974

Antifer Ameghino, 1889

Especie tipo. Antifer ultra (Ameghino, 1888).
Comentarios. Actualmente se reconocen para Antifer dos especies fundadas a partir de astas incompletas. A. ultra (Ameghino, 1888) se registra en sedimentos del Pleistoceno medio y tardío en las provincias de Santa Fe, Buenos Aires y Entre Ríos, Argentina (Castellanos, 1945; Menegaz, 2000; Alcaraz et al., 2005). A. ensenadensis (Ameghino, 1888), la de mayor robustez, se encuentra sin duda en sedimentos del Pleistoceno temprano (Piso/Edad Ensenadense) de la Provincia de Buenos Aires, Argentina (Menegaz, 2000). Es importante mencionar que Castellanos (1945), citó para la Provincia de Santa Fe a $A$. ensenadensis a base del ejemplar MUFyCA $\mathrm{N}^{\mathrm{o}} 16$, procedente de sedimentos "belgranenses" en el sentido de Ameghino (1889); sin embargo, la antigüedad de los sedimentos portadores no es clara para el lugar de extracción y el material debe ser revisado nuevamente. La existencia de dos especies diferentes de Antifer estuvo en duda durante mucho tiempo, dado que fueron propuestas sobre bases morfológicas y morfométricas escasamente fundamentadas (Kraglievich, 1932; Menegaz, 2000). Desde una perspectiva paleoecológica, Antifer estaría asociado a ambientes arbustivos y habría desarrollado un hábito trófico ramoneador (Menégaz \& Ortiz Jaureguizar, 1995).

\section{Antifer ensenadensis (Ameghino, 1888)}

(Figura 2A)

Material referido. CTES-PZ 7486, parte de un asta derecha que conservó el pedúnculo, sin roseta, y su bifurcación principal.

Procedencia geográfica y estratigráfica. Localidad de Lavalle (Departamento de Lavalle), ubicada a $180 \mathrm{~km}$ al sur de la ciudad de Corrientes, Formación Toropí, Pleistoceno tardío. Descripción. El ejemplar CTES-PZ 7486 es muy robusto, conservó el pedúnculo completo sin roseta y parte de la

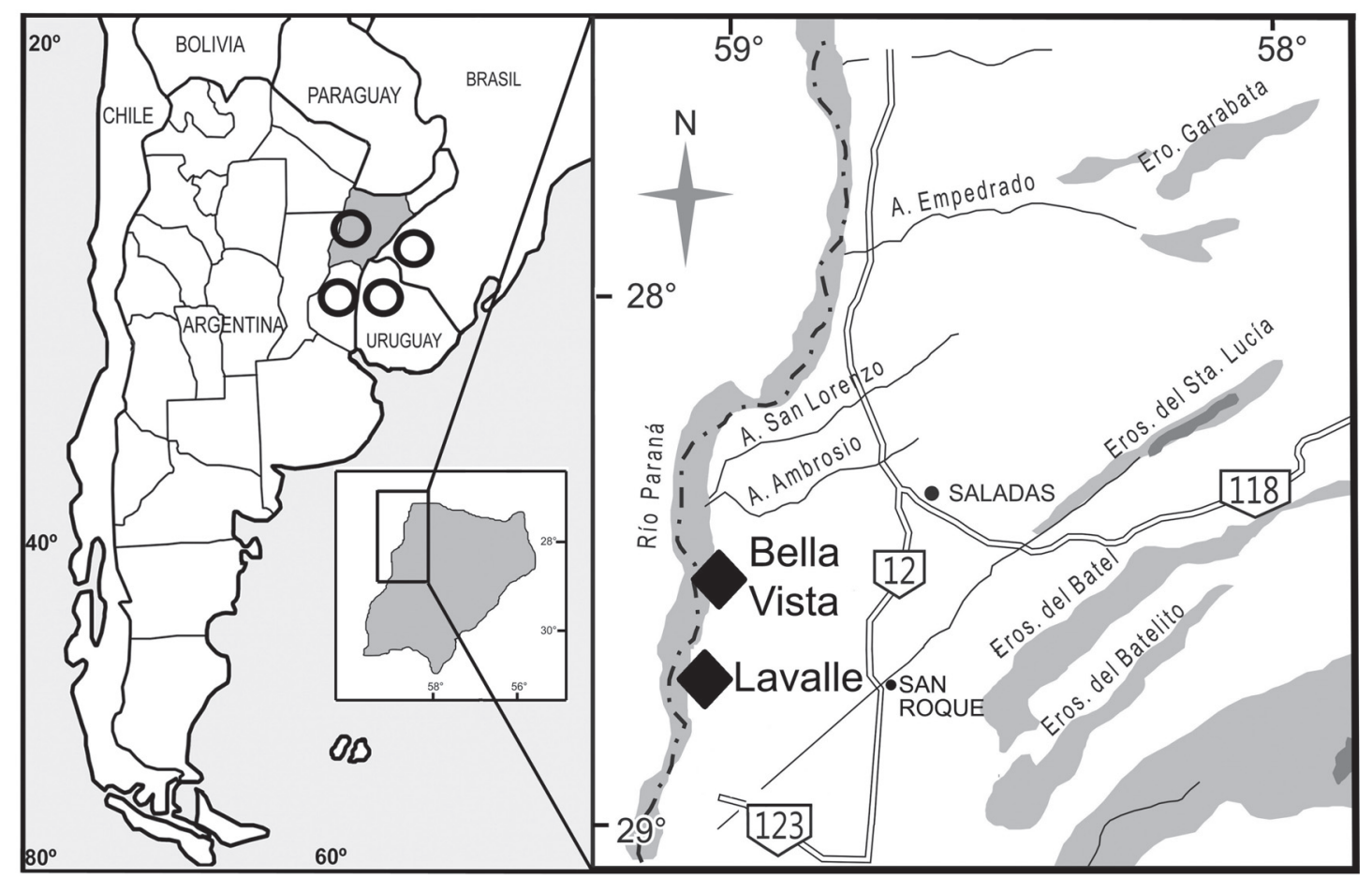

Figura 1. Mapa mostrando las localidades paleontológicas de la Provincia de Corrientes y las formaciones pleistocenas de áreas geográficas cercanas.

Figure 1. Map showing the paleontological localities of Corrientes Province and the Pleistocenic formations in close geographic areas. 
bifurcación principal. El pedúnculo es columnar muy desarrollado, cilíndrico, recto y largo comprimiéndose transversalmente a nivel de la bifurcación principal. Presenta una base ancha y circular perfecta que es completamente plana y recta. El borde anterior del pedúnculo es algo cóncavo, mientras que el posterior es bien recto y ancho. La garceta es grácil, se encuentra rota en su extremo, pero por la porción conservada se aprecia que era simple. La rama posterior, de la que sólo se conservó una pequeña porción, es ensanchada transversalmente y en la fractura que presenta se observa su interior hueco. La cara externa es convexa y la interna suavemente cóncava, ambas están recorridas por crestas longitudinales apenas marcadas en este ejemplar. Si bien se conservó sólo una porción de las ramas de la bifurcación principal, puede verse en vista lateral que estas tienen una leve inclinación hacia el plano sagital.

\section{Antifer ultra (Ameghino, 1888)}

(Figuras 2B-D)

Material referido. CTES-PZ 7160, asta izquierda incompleta; CTES-PZ 7290, asta izquierda incompleta; CTES-PZ 7367, asta derecha casi completa.

Procedencia geográfica y estratigráfica. Localidad de Lavalle (Departamento de Lavalle), ubicada a $180 \mathrm{~km}$ al sur de la ciudad de Corrientes, Formación Toropí, Pleistoceno tardío. Descripción. El ejemplar CTES-PZ 7367 es un asta pequeña y grácil, correspondiente al volteo de un individuo juvenil que consta de tres ramas representadas por la garceta y la rama posterior o vara bifurcada. La base del asta es circular, angosta e inclinada hacia delante y medialmente, con la presencia de una pequeña protuberancia $\mathrm{o}$ almohadilla central sobresaliente en la zona de desprendimiento del asta (carácter presente en muchos de los ejemplares de esta especie). Por encima de la base, se encuentra la roseta que no se conservó completa observándose sólo algunas pequeñas excrecencias óseas de ella. Por encima de ella, el pedúnculo está bien desarrollado, comienza basalmente cilíndrico y se va ensanchando en sentido transversal hacia su extremo distal, alcanzando su máximo ensanchamiento a nivel de la bifurcación principal. Este, está constituido por un borde anterior marcadamente cóncavo y un borde posterior convexo, que otorgan al pedúnculo, en sección, la característica morfología de "V". La garceta es simple y grácil, se encuentra implantada en un ángulo obtuso respecto al pedúnculo y agudo en cuanto a la rama posterior. En la cara interna del asta se observa un canal bien marcado que se origina en la base del pedúnculo y cuya mayor profundidad se da a nivel de la garceta; por su parte, la cara externa del asta muestra a nivel de la bifurcación principal, otra fosa profunda que se extiende hasta la mitad del recorrido de la garceta. La vara o rama posterior es delgada y a cierta distancia se bifurca dando origen a una rama anterior y otra posterior, que forman un ángulo agudo entre sí. En la cara externa de la vara y a nivel de su bifurcación, se dispone una fosa bien profunda de forma triangular y base superior, ya mencionada en otros ejemplares de Antifer ultra (Alcaraz et al., 2005). Tanto la cara interna como externa del asta, presentan en toda su superficie estrías y surcos longitudinales bien marcados. Todas las ramas del asta están fuertemente inclinadas hacia el plano sagital. El asta CTES-PZ 7290 fue estudiada por Alcaraz \& Zurita (2004), en consecuencia aquí sólo se va a tratar su status taxonómico, ya que en ese momento fue atribuida a Epieuryceros cf. E. proximus. Sin embargo, su reestudio permitió observar que presenta: la base con los mismos rasgos morfológicos que el ejemplar CTESPZ 7367, la característica morfología del pedúnculo en "V" en sección, el aplastamiento de la rama posterior en sentido transversal, además de un tamaño y robustez semejante, menores a los de las astas de la otra especie, permitiendo así su inclusión en A. ultra. El ejemplar CTES-PZ 7160 también ha sido anteriormente descripto y estudiado (Zurita \& Lutz, 2002). El análisis de nuevos materiales y su comparación morfológica con ellos considerando los atributos mencionados más arriba, demostraron que el espécimen corresponde a un individuo de $A$. ultra.

Mazama Rafinesque, 1817

Especie tipo. Mazama pita Rafinesque, 1817.

\section{cf. Mazama sp.}

(Figura 3A)

Material referido. CTES-PZ 7485, porción proximal de asta con la roseta parcial.

Procedencia geográfica y estratigráfica. Arroyo Toropí, Bella Vista (Departamento de Bella Vista) Provincia de Corrientes, Formación Yupoí, Pleistoceno tardío.

Comentarios. El género Mazama se caracteriza por la presencia de astas simples formadas por una sola rama de forma puntiaguda. Actualmente tiene una amplia distribución en la Argentina, incluyendo tres especies: Mazama gouazoubira Fischer, 1814 (corzuela parda); M. nana Hensel, 1872 (corzuela enana) y M. americana (Erxleben, 1777) (corzuela colorada). El género viviente Pudu Gray, 1852, presenta igualmente astas simples y morfológicamente similares a las de Mazama, al menos en la zona de la roseta. Sin embargo, tanto su distribución actual exclusivamente andina ( $\sin$ reportes conocidos en áreas extraandinas), como la ausencia de registro paleontológico o arqueológico (Guerin \& Faure, 2009), indican que muy probablemente el género $P u d u$ se haya diferenciado en esos ámbitos y tenga una fuerte restricción en cuanto a sus requerimientos. Esta situación, apoyaría la hipótesis de que el material aquí descripto pertenezca a Mazama y no a Pudu, en tanto que las especies de Mazama viven en diferentes tipos de bosques, montes, hasta selvas subtropicales con vegetación densa, donde se alimentan de frutos, brotes y ramas tiernas de arbustos y árboles (Canevari \& Vaccaro, 2007). El registro paleontológico de este género en la Argentina, se encuentra restringido al sector centrooriental (región Pampeana) y todas las citas previas provienen de sedimentos holocenos (Piso/Edad Platense) (Castellanos, 1924). Según Tonni (2009) durante el Holoceno, a partir de los ca. 7.000 años AP comenzó un evento cálido y húmedo representado por la expansión de fauna subtropical en distintos sectores de la Provincia de Buenos Aires. Fuera del territorio 


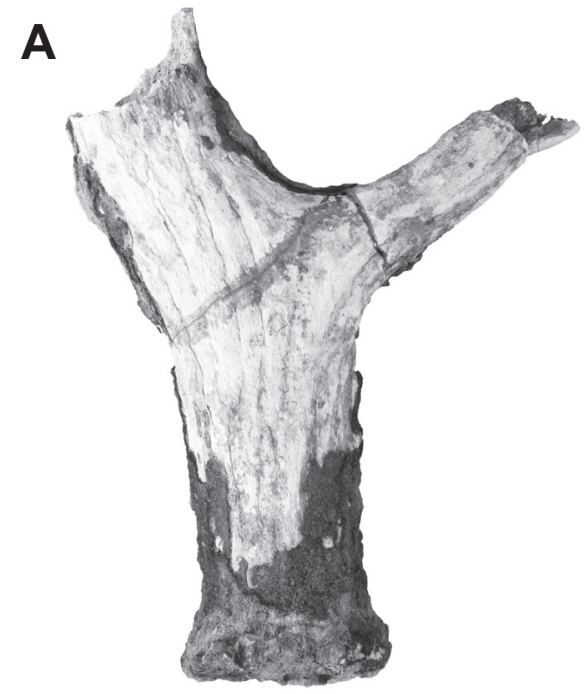

\section{B}
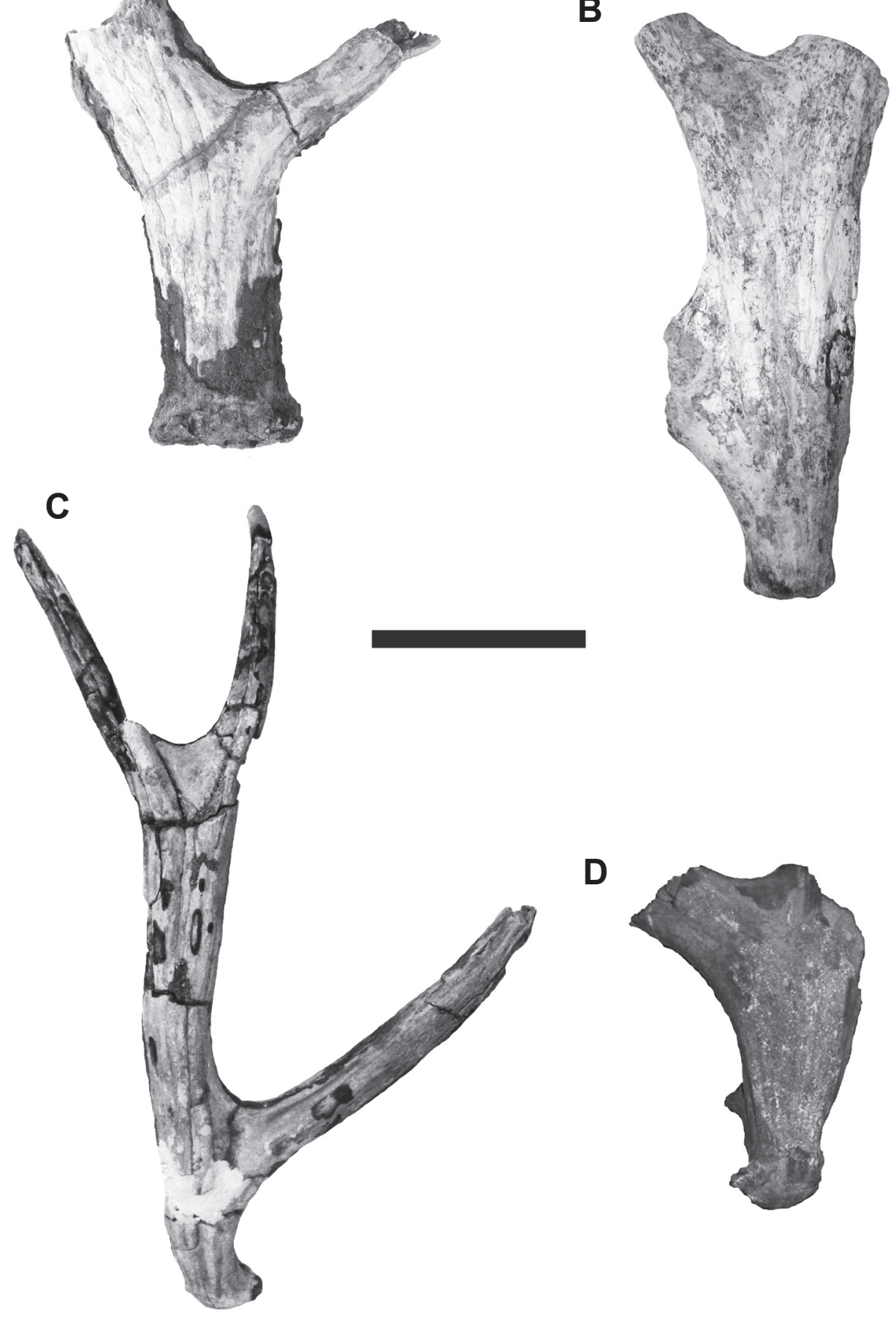

Figura 2. Porciones de astas de Antifer en vista externa. A, A. ensenadensis, CTES-PZ 7486, asta derecha. B, A. ultra, CTESPZ 7160, asta izquierda; C, CTES-PZ 7367, asta derecha; D, CTES-PZ 7290, asta izquierda. Escala $=10 \mathrm{~cm}$.

Figure 2. Partial antlers of Antifer in external view. A, A. ensenadensis, CTES-PZ 7486, right antler. B, A. ultra, CTES-PZ 7160, left antler; C, CTES-PZ 7367, right antler; D, CTES-PZ 7290, left antler. Scale bar $=10 \mathrm{~cm}$.

argentino, se reportó la presencia de $M$. americana y $M$. gouazoubira en sedimentos del Pleistoceno tardío-Holoceno temprano del noreste de Brasil, en el Parque Nacional Serra da Capibara (Guérin \& Faure, 2009), y de restos asignados a cf. Mazama sp. en el norte de Uruguay (Formación Sopas) (Corona \& Ubilla, 2010).

\section{Morenelaphus Carette, 1922}

Especie tipo. Morenelaphus brachyceros (Ameghino, 1888).

Comentarios. El género extinto Morenelaphus cuenta con dos especies, M. lujanensis (Ameghino, 1888) y M. brachyceros 
(H. Gervais \& Ameghino, 1880). Ambas están citadas en la Argentina para las provincias de Buenos Aires y Santa $\mathrm{Fe}$ (Menegaz, 2000), además de Entre Ríos (Ferrero et al., 2007; Ferrero, 2009). La primera de ellas, se encuentra restringida a sedimentos de antigüedad Pleistoceno tardío (Piso/Edad Lujanense) (Menegaz, 2000; Ferrero et al., 2007), y la segunda presente en el Pleistoceno medio y tardío (Pisos/Edades Bonaerense y Lujanense) (Menegaz, 2000; Ferrero, 2009).

\section{Morenelaphus lujanensis (Ameghino, 1888)}

(Figura 3B)

Material referido. CTES-PZ 7369, asta derecha completa. Procedencia geográfica y estratigráfica. Arroyo Toropí, Bella Vista (Departamento de Bella Vista) Provincia de Corrientes. Formación Toropí. Pleistoceno tardío.

Comentarios. Scillato-Yané et al. (1998) asignaron el ejemplar CTES-PZ 7369 a Morenelaphus brachyceros (Gervais \& Ameghino 1880), sin hacer referencia al análisis anatómico del fósil. En este sentido, la curvatura de su rama principal, la ubicación y disposición de sus candiles $2^{\circ}$ y $3^{\circ}, y$ de ellos respecto a la garceta, el mayor tamaño y la gracilidad que presenta el asta en comparación con la otra especie (Alcaraz, 2010) permiten aquí su reasignación a M. lujanensis. Desde un punto de vista paleoecológico a las especies de Morenelaphus se las interpreta como ramoneadoras, al igual que Antifer (Menégaz \& Ortiz Jaureguizar, 1995).

\section{Morenelaphus sp.}

Material referido. CTES-PZ 1605, porción del pedúnculo de un asta derecha.

Procedencia geográfica y estratigráfica. Arroyo Toropí, Bella Vista (Departamento de Bella Vista) Provincia de Corrientes, Formación Yupoí, Pleistoceno tardío.

Comentarios. este material fue determinado previamente por Álvarez (1974) como Blastocerus Gray, 1850, sin embargo su restudio permite reasignarlo aquí a Morenelaphus sp. En el ejemplar se observan los caracteres morfológicos del género, es decir el pedúnculo corto y grácil, cóncavo del lado externo y plano en su lado interno, y de ninguna manera, este pedúnculo podría alcanzar el tamaño mayor, la morfología y la robustez de los ejemplares de Blastocerus, por lo que se descarta esa asignación. De esta manera, se sostiene lo propuesto por Menegaz (2000), quien establece que no existen registros de Blastocerus previos al Holoceno en Argentina.

\section{Hippocamelus Leuckart, 1816}

Especie tipo. Hippocamelus dubius Leuckart, 1816.

Hippocamelus sulcatus (Ameghino, 1888) (Figura 3C)

Material referido. CTES-PZ 7494, parte del cráneo con ambas astas; porción de la hemimandíbula derecha con la dentición premolar y molar completa, fragmento del maxilar izquierdo con los M1-M3, vértebras cervicales, vértebra dorsal, fragmentos de costillas aisladas, escápula izquierda incompleta, húmero izquierdo sin epífisis proximal, ambas ulnas, ambos radios, carpo izquierdo, ambos metacarpos, tibia izquierda, hueso maleolar, astrágalo, calcáneo, gran cuneiforme y cuboideo-navicular izquierdos, metatarso izquierdo, 3 falanges I, falange II y falange III (Alcaraz, 2010).

Procedencia geográfica y estratigráfica. Arroyo Toropí, Bella Vista (Departamento de Bella Vista) Provincia de Corrientes, Formación Toropí, Pleistoceno tardío.

Comentarios. El género Hippocamelus cuenta con tres especies, dos vivientes, H. antisensis (d'Orbigny, 1834) (taruca) e H. bisulcus (Molina, 1782) (huemul), ambas con una distribución andina. La primera de estas especies se alimenta de plantas suculentas, gramíneas, arbustos e incluso líquenes, mientras que $H$. bisulcus, lo hace principalmente de hierbas, gramíneas, arbustos y hojas de árboles (Canevari \& Vaccaro, 2007). La tercera, H. sulcatus, es extinta y sus registros en la Argentina se restringen a las provincias de Buenos Aires en sedimentos del Pleistoceno medio (Piso/Edad Bonaerense) (Menegaz, 2000), y de Corrientes durante el Pleistoceno tardío (Piso/Edad Lujanense) (Alcaraz, 2010). En Argentina, para ambas regiones donde fue hallado $H$. sulcatus, se infieren ambientes con características geomorfológicas distintas al del actual hábitat de las especies de ese género. Fuera del territorio argentino las citas para $H$. sulcatus se limitan al oeste de la República de Uruguay (departamentos de Soriano y Río Negro) a sedimentos referidos al Pleistoceno (Kraglievich, 1932). Como se mencionó anteriormente, en esta contribución no se da una descripción detallada del ejemplar ya que forma parte de un trabajo más extenso y específico, aún en preparación. Sin embargo su inclusión en este estudio, radica en la importancia de su aporte en la caracterización de la diversidad de cérvidos presentes en la Provincia de Corrientes durante el Pleistoceno.

\section{DISCUSIÓN}

Del conjunto de materiales analizados y de su identificación taxonómica, llama la atención la alta diversidad de Cervidae para las dos unidades litoestratigráficas sucesivas, ya que en la Formación Toropí se registraron Morenelaphus lujanensis, Antifer ultra, A. ensenadensis e Hippocamelus sulcatus; mientras que en la Formación Yupoí, se reconocieron a cf. Mazama sp. y Morenelaphus sp. Resulta llamativa tal riqueza de especies para un área restringida de afloramientos y un corto lapso temporal, más aún, considerando comparativamente la diversidad de cérvidos presentes durante el Pleistoceno en otras áreas geográficamente cercanas (ver Tabla 1). En este contexto, la Formación Toropí presenta una diversidad similar a la hallada en la Formación Sopas, sin embargo tanto la antigüedad de los sedimentos portadores como las especies presentes en ambas formaciones son diferentes.

La Formación Sopas exhibe particularidades de connotación cronológica y biogeográfica, en tanto en general se acepta para ella una antigüedad correspondiente al Pleistoceno tardío, sobre la base de fechados carbónicos (Martínez \& Ubilla, 
A

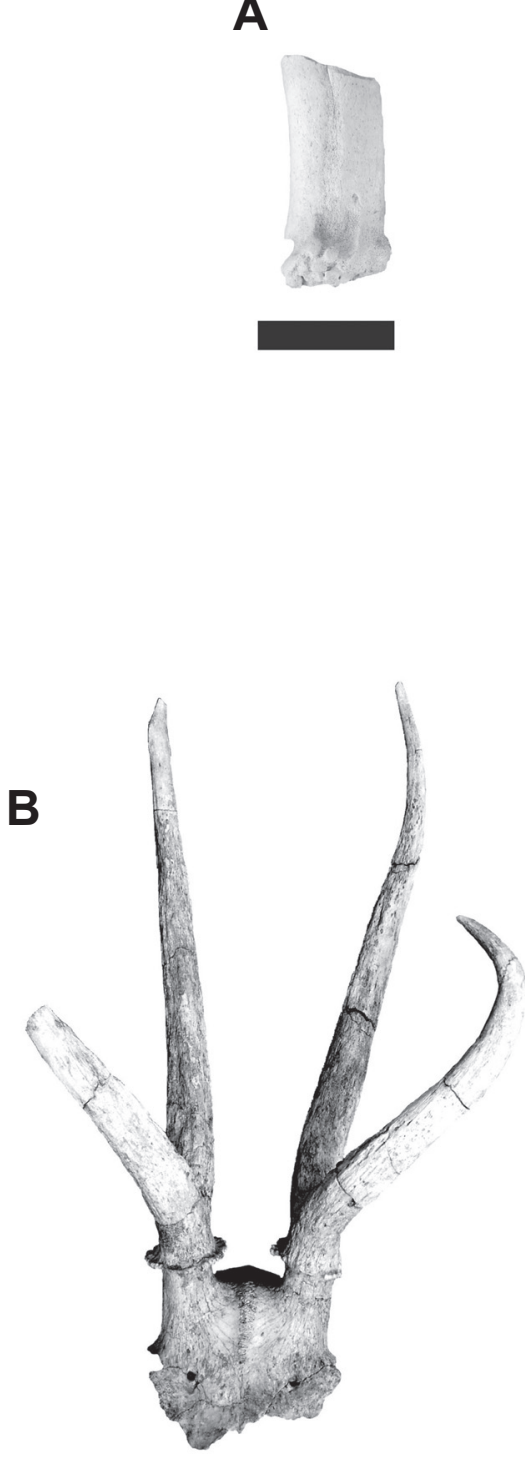

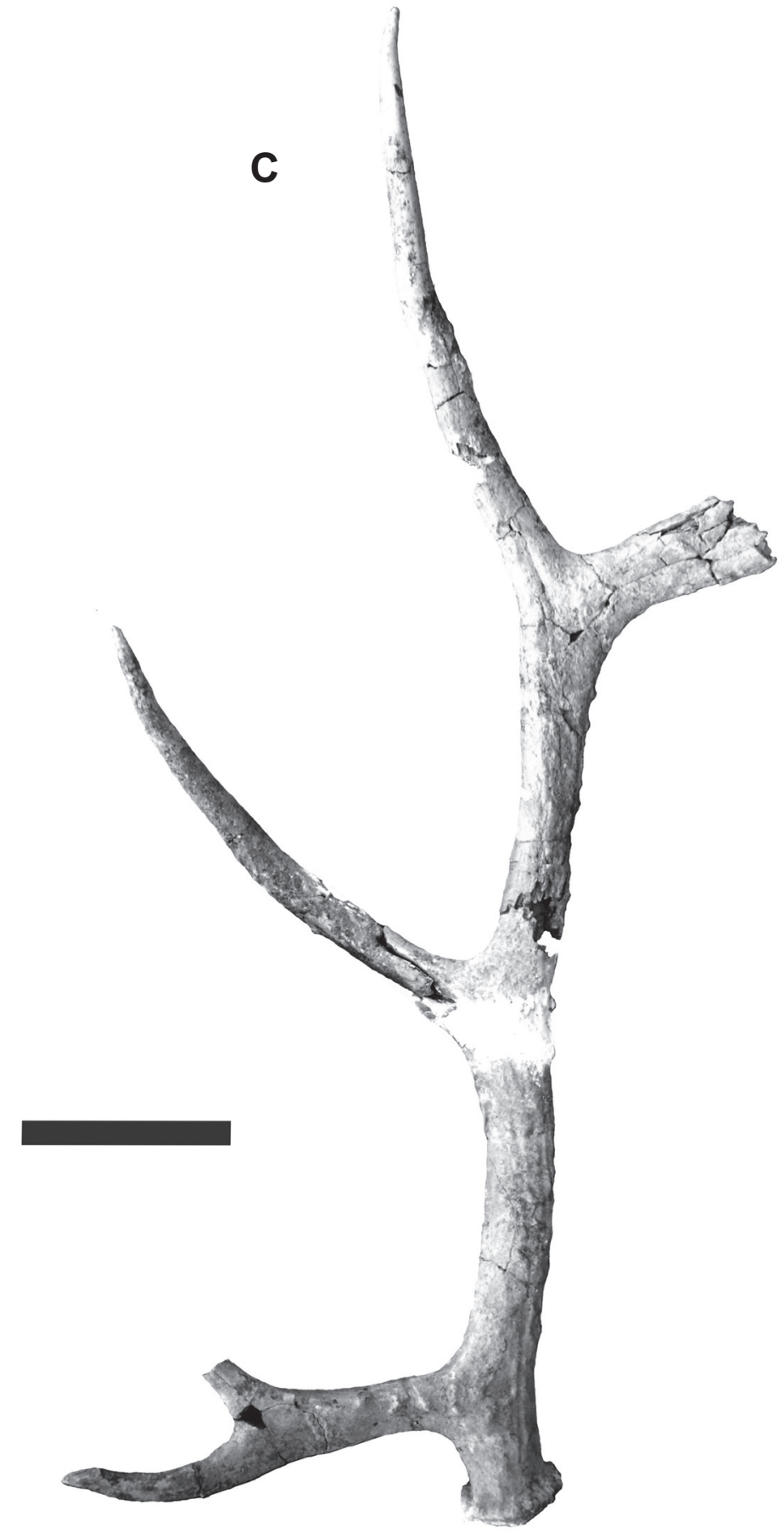

Figura 3. Astas. A, cf. Mazama, CTES-PZ 7485, porción proximal. B, Hippocamelus sulcatus, CTES-PZ 7494, parte del cráneo con ambas astas. C, Morenelaphus lujanensis, CTES-PZ 7369, asta derecha completa. Escalas: A=2 cm; B-C = $10 \mathrm{~cm}$.

Figure 3. Antlers. A, cf. Mazama, CTES-PZ 7485, proximal portion. B, Hippocamelus sulcatus, CTES-PZ 7494, partial skull with both antlers. C, Morenelaphus lujanensis, CTES-PZ 7369, complete right antler. Scale bars: A=2 cm; B-C = $10 \mathrm{~cm}$.

2004). No obstante, nuevas técnicas de dataciones aplicadas recientemente (TL/OSL) dieron por un lado resultados que lo apoyan, y por el otro edades entre los $c a .180 .000 \pm 20.000 \mathrm{y}$ $360.000 \pm 40.000$ años AP, que se corresponden con la etapa tardía del Pleistoceno medio (Martínez \& Ubilla, 2004). En cuanto a las especies presentes en el Pleistoceno de Uruguay, tanto Ozotoceros bezoarticus (Linnaeus, 1758) como Paraceros fragilis (Ameghino, 1888) no se registran en la Provincia de
Corrientes (ver Tabla 1). Al respecto, las citas conocidas de $P$. fragilis en la Argentina se encuentran restringidas a la Provincia de Buenos Aires (Menegaz, 2000), mientras que $O$. bezoarticus aparece con seguridad en el Holoceno, siendo dudosos los reportes hechos hasta el momento para el Pleistoceno de la Argentina (Alcaraz, obs. pers.).

Es importante resaltar en la Formación Toropí, la presencia de dos formas de gran tamaño como Antifer ultra y 
A. ensenadensis, y una de mediano a gran porte como Morenelaphus lujanensis. Según Alcaraz (2010), ambos géneros tuvieron una amplia distribución geográfica en la Argentina ya que se registran desde los $25^{\circ}-38^{\circ}$ de latitud sur. Esto evidencia la gran plasticidad adaptativa para ocupar una gran variedad de ambientes posiblemente como respuesta a la disponibilidad de recursos. La presencia de estas especies geográfica y estratigráficamente asociadas no necesariamente tuvo que haber sido sincrónica, más aun considerando que la coexistencia de especies con tales características corporales, no se observa actualmente entre las formas vivientes. Desde un punto de vista paleoclimático-ambiental, es interesante mencionar para la secuencia formaciones Toropí/Yupoí y la Formación Sopas, el posible desarrollo de un escenario dinámico y propio de zonas ecotonales afectadas fácilmente por el cambio de las variables paleoclimáticas más relevantes (e.g. temperatura y humedad), probablemente favorecido por condiciones climáticas benignas propias de ciertas latitudes de América del Sur (Francia et al., 2012a; Ubilla, 1996). En particular, para el Pleistoceno tardío de la Provincia de Corrientes a base de recientes evidencias paleobotánicas se infiere el desarrollo de ambientes abiertos de pastizales con algunas palmeras y otros árboles y arbustos presentes sobre las márgenes de un sistema fluvial, en un clima relativamente templado-cálido (Erra et al., 2011). Esta comunidad vegetal mixta refleja una afinidad biogeográfica entre la llanura Chaco-pampeana y las regiones intertropicales, relacionadas por las frecuentes fluctuaciones climático-ambientales dadas durante el Pleistoceno (Erra et al., en prensa).

\section{CONCLUSIONES}

Se reporta por primera vez a Morenelaphus lujanensis en la Provincia de Corrientes, constituyendo además, el segundo registro para la región Mesopotámica (ver Ferrero, 2009). Los materiales anteriormente asignados a Antifer aff A. ensenadensis por Zurita \& Lutz (2002) y a Epieuryceros cf. E. proximus por Alcaraz \& Zurita (2004) son considerados aquí pertenecientes a Antifer ultra. Por otro lado, se descartó la cita de Blastocerus en la Formación Toropí (Alcaraz, 2010), ese material corresponde a Morenelaphus sp. Se informa la presencia de cf. Mazama sp. en sedimentos del Pleistoceno tardío, lo que apoya su registro en el territorio de la Argentina con anterioridad al Holoceno.

Paleozoogeográficamente se extiende la corología de Antifer ensenadensis hacia el noreste de la Argentina, al tiempo que desde una perspectiva bioestratigráfica, se amplía su biocrón hasta el Piso/Edad Lujanense. De esta manera, constituye el registro más septentrional dentro del territorio argentino. Desde un enfoque paleoecológico, la gran diversidad de cérvidos presentes en el Pleistoceno tardío de la Provincia de Corrientes podría deberse a la plasticidad del grupo para adaptarse a condiciones ambientales variables. En parte, esto puede especularse por la presencia de Antifer y de Morenelaphus, dos géneros con amplia distribución latitudinal y que ocuparon seguramente una importante diversidad de ambientes (Alcaraz, 2010).

Para el Pleistoceno tardío de Corrientes, se observa una mayor diversidad específica en la Formación Toropí, sólo comparable con la acontecida durante el Pleistoceno en la Formación Sopas de Uruguay. Sin embargo, algunas de las especies presentes y la antigüedad de los sedimentos portadores, no son análogas en ambas formaciones. Más aún, si consideramos la asociación de ambas unidades litoestratigráficas (formaciones Toropí/Yupoí) como postula Iriondo $(1996,2010)$ y teniendo en cuenta el acotado lapso de tiempo representado para dicha unidad, sería todavía más relevante la alta diversidad existente, reuniendo para un intervalo temporal de aproximadamente 20.000 años a los siguientes taxones: Morenelaphus lujanensis, Morenelaphus sp., Antifer ultra, A. ensenadensis, Hippocamelus sulcatus y cf. Mazama.

\section{AGRADECIMIENTOS}

A E.P. Tonni y A.A. Carlini, por sus comentarios y aportes, que ayudaron a enriquecer de manera significativa el contenido del manuscrito. A M.C. de Castro por su colaboración en la traducción del abstract.

Tabla 1. Diversidad de Cervidae procedentes de distintas formaciones del Pleistoceno tardío de áreas geográficas cercanas de la Argentina, Brasil y Uruguay. La línea punteada representa la unificación litoestratigráfica postulada por Iriondo (1996, 2010).

Table 1. Diversity of Cervidae from different formations of the late Pleistocene in close geographic areas of Argentina, Brazil, and Uruguay. The dotted line represents the litostratigraphic unification proposed by Iriondo (1996, 2010).

\begin{tabular}{|c|c|c|c|c|c|}
\hline \multicolumn{4}{|c|}{ ARGENTINA } & \multirow{3}{*}{$\begin{array}{l}\text { URUGUAY } \\
\text { Fm. Sopas }\end{array}$} & \multirow{3}{*}{$\begin{array}{c}\text { BRASIL } \\
\text { Fm. Touro Passo }\end{array}$} \\
\hline \multicolumn{2}{|c|}{ Provincia de Corrientes } & \multicolumn{2}{|c|}{ Provincia de Entre Ríos } & & \\
\hline Fm. Toropí & Fm. Yupoí & Fm. Tezanos Pinto & Fm. El Palmar & & \\
\hline \multirow{3}{*}{$\begin{array}{c}\text { Morenelaphus } \\
\text { lujanensis }\end{array}$} & Morenelaphus sp. & \multirow[b]{3}{*}{$\begin{array}{c}\text { Morenelaphus } \\
\text { brachyceros }\end{array}$} & \multirow[b]{2}{*}{$\begin{array}{c}\text { Morenelaphus cf. } \\
\quad \text { lujanensis }\end{array}$} & & \multirow[t]{3}{*}{ Morenelaphus sp. } \\
\hline & & & & $\begin{array}{l}\text { Morenelaphus } \\
\text { lujanensis }\end{array}$ & \\
\hline & & & \multirow{8}{*}{ Antifer sp. } & $\begin{array}{c}\text { Morenelaphus } \\
\text { brachyceros }\end{array}$ & \\
\hline \multirow{7}{*}{$\begin{array}{c}\text { Antifer ultra } \\
\text { Antifer ensenadensis } \\
\text { Hippocamelus sulcatus }\end{array}$} & \multirow{7}{*}{ cf. Mazama } & \multirow{7}{*}{ Antifer ultra } & & & \multirow[t]{7}{*}{ Antifer sp. } \\
\hline & & & & Antifer ultra & \\
\hline & & & & & \\
\hline & & & & & \\
\hline & & & & cf. Mazama & \\
\hline & & & & $\begin{array}{c}\text { Ozotoceros aff. } O \text {. } \\
\text { bezoarticus }\end{array}$ & \\
\hline & & & & Paraceros fragilis & \\
\hline
\end{tabular}




\section{REFERENCIAS}

Aguilera S. \& Orangel A. 2004. Tesoros paleontológicos de Venezuela. Urumaco, Patrimonio Nacional de la Humanidad. Caracas, Universidad Nacional Experimental Francisco de Miranda, $148 \mathrm{p}$

Ameghino, F. 1889. Contribución al conocimiento de los mamíferos fósiles de la República Argentina. Actas de la Academia Nacional de Ciencias de Córdoba, 6:1-1027.

Ameghino, F. 1902. Nota sobre algunos mamíferos fósiles nuevos o poco conocidos del Valle de Tarija. Anales del Museo Nacional de Buenos Aires, 8:1-250 p.

Alcaraz, M.A. 2010. Sistemática de los cérvidos (Mammalia, Artiodactyla) del Pleistoceno de las áreas extraandinas de Argentina. Universidad Nacional de La Plata, Tesis Doctoral, 317 p.

Alcaraz, M.A. \& Carlini, A.A. 2003. Los cérvidos (Mammalia, Artiodactyla) de las formaciones Toropí y Yupoí (Pleistoceno medio-tardío) de la Provincia de Corrientes, Argentina. Comunicaciones Cientificas y Tecnológicas de la Universidad Nacional del Nordeste, Corrientes. Available at http//www.unne. edu. ar/; accessed on 10/03/2009.

Alcaraz, M.A; Ferrero, S.B. \& Noriega J.I. 2005. Primer registro de Antifer ultra Ameghino, 1889 (Artiodactyla: Cervidae) en el Pleistoceno de Entre Ríos. Revista del Instituto Superior de Correlación Geología, 14:65-70.

Alcaraz, M.A. \& Francia, A. 2010. Diversidad de Cérvidos (Mammalia, Artiodactyla) durante el Pleistoceno de la Provincia de Corrientes, Argentina. In: JORNADAS ARGENTINAS DE MASTOZOOLOGÍA, 23, 2010. Libro de resúmenes, Bahía Blanca, Universidad Nacional del Sur, p. 41.

Alcaraz, M.A. \& Zurita, A.E. 2004. Nuevos registros de cérvidos poco conocidos: Epieuryceros cf. proximus Castellanos y Antifer sp. (Mammalia, Artiodactyla, Cervidae). Revista del Museo Argentino de Ciencias Naturales, 6:41-48.

Álvarez, B.B. 1974. Los Mamíferos fósiles del Cuaternario de Bella Vista, Corrientes (Argentina). Ameghiniana, 11:295-311.

Bagnalasta, M.B. 1980. Algunas consideraciones sobre la validez del género Epieuryceros Ameghino, 1889. In: CONGRESOARGENTINO PALEONTOLOGÍA, 2/CONGRESOLATINOAMERICANO, 1, 1978. Libro de Actas, Buenos Aires, 3:183-192.

Bombin, M. 1976. Modelo Paleoecológico-evolutivo para o Neoquaternário da regiao da Campanha- Oeste do Rio Grande do Sul (Brasil). A Formação Touro Passo, seu conteúdo fossilífero e a pedogénese pós-deposicional. Comunicações do Museu de Ciências da PUCRGS, 15:1-90.

Canevari, M. \& Vaccaro, O. 2007. Guía de Mamiferos del Sur de América del Sur. Buenos Aires, Ed. L.O.L.A., 413 p.

Carlini, A.A. \& Tonni, E.P. 2000. Mamíferos Fósiles del Paraguay. Buenos Aires, Cooperación Técnica Paraguayo-Alemana, 108 p. (Proyecto Sistema Ambiental del Chaco-Proyecto Sistema Ambiental Región Oriental).

Casamiquela, R. 1976. Los vertebrados fósiles de Tagua-Tagua, Chile. In: CONGRESO GEOLÓGICO CHILENO, 1, 1976. Actas, Santiago, p. C87-C102.

Casamiquela, R. 1984. Critical catalogue of some Chilean fósil vertebrales. I. The Deers: Complementary considerations on Antifer (Antifer niemeyeri n. sp.), the Pleistocene Giant Deer. Quaternary of South America and Antartic Peninsula, 2:41-50.

Casamiquela, R. 1999. The vertebrate record of Chile. Quaternary of South America and Antarctic Peninsula, 7:91-107.

Castellanos, A. 1924. Ciervos vivientes y extinguidos de la República Argentina (Nota preliminar). Revista de la Universidad de Córdoba, 11:111-134.
Castellanos, A. 1945. Nota sobre algunas cornamentas de ciervos de los géneros Epieuryceros, Antifer y Paraceros y descripción de la de Epieuryceros proximus n. sp.. Publicaciones del Instituto de Fisiografia y Geología de la facultad de Ciencias FísicoQuímicas y Naturales aplicadas a la Industria de la Universidad Nacional del Litoral, 24:5-23.

Corona, A. \& Ubilla, M. 2010. Cf. Mazama sp. (Mammalia, Cervidae) en el Pleistoceno tardío del norte de Uruguay (Formación Sopas). In: CONGRESO URUGUAYO DE GEOLOGÍA, 6, 2010. Actas, Montevideo, p. 18-19.

Churcher, C.S. 1962. Odocoileus salinae and Mazama sp. from Talara tar seeps, Perú. Life Science Contributions, 57:1-27.

Emmons, L.H. 1999. Mamíferos de los bosques húmedos de América tropical. Santa Cruz de la Sierra, Editorial F.A.N., 298 p.

Erra, G; Osterrieth, M.; Zurita, A.E.; Francia, A. \& Carlini, A.A. 2011. Paleoambientes alternates en el Pleistoceno de Corrientes Una perspectiva fotolítica. In: JORNADAS ARGENTINAS DE BOTÁNICA, 33, 2011. Actas, Misiones, p. 253.

Erra, G.; Osterrieth, M.; Zurita, A.E.; Francia, A. \& Carlini, A.A. (en prensa). Paleoenvironment of the Toropí Formation (Upper Pleistocene), Corrientes province Mesopotamian Region, Argentina): a phytolith approach. Quaternary International. doi:10.1016/j.quaint.2012.08.2110

Ferrero, B.S. 2009. Mamíferos del Cuaternario de la provincia de Entre Ríos, Argentina: Diversidad y evolución. Aspectos bioestratigráficos y paleozoogeográficos de una fauna particular. Universidad Nacional de La Plata, Tesis Doctoral, 425 p.

Ferrero, B.S.; Brandoni, D.; Noriega, J.I. \& Carlini, A.A. 2007. Mamíferos de la Formación El Palmar (Pleistoceno tardío) de la provincia de Entre Ríos, Argentina. Revista del Museo Argentino de Ciencias Naturales, 9:109-117.

Francia A.; Carlini, A.A.; Zurita, A.E.; Miño-Boilini, A.R. \& Kruck, W. 2012b. Cronología de las unidades litoestratigráficas aflorantes en el Arroyo Toropí, Provincia de Corrientes y los registros paleofaunísticos. In: REUNIÓN DE COMUNICACIONES CIENTÍFICAS Y TECNOLÓGICAS, 2012. Libro de resúmenes on-line, Corrientes, versión sin paginación CB-033 (http//www. unne.edu.ar).

Francia, A.; Carlini, A.A.; Zurita, A.E. \& Verzi, D.H. 2012a. Galea (Rodentia, Caviidae) in the late Pleistocene of Corrientes Province (Argentina): taxonomic and paleobiogeographic implications Neues Jahrbuch für Geologie und Paläontologie Abhandlungen, 266:173-184. doi:10.1127/00777749/2012/0292

Gervais, H. \& Ameghino, F. 1880. Los mamíferos fósiles de la América de Sud. París-Buenos Aires, Sabih e Igon, 225 p.

Guérin, C. \& Faure, M. 2009. Les Cervidae (Mammalia, Artiodcatyla) du Pléistocène supérieur - Holocène ancien de la région du Parc Nacional Serra da capibara (Piauí, Brasil). Geobios, 42:169-195. doi:10.1016/j.geobios.2008.06.004

Herbst, R. \& Álvarez, B.B. 1977. Nota sobre dos Formaciones del Cuaternario de Corrientes, Republica Argentina. Facultad de Ciencias Exactas y Naturales y Agrimensura, 1:7-17.

Herbst, R. \& Santa Cruz, J.N. 1999. Mapa litoestratigráfico de la Provincia de Corrientes. D'Orbignyana 2:1-69.

Herreros de Lartundo, J. \& Sielfeld Kowald, W. 2011. La Taruca, Huemul del norte. Chile, Ministerio del Medioambiente, 64 p.

Hoffstetter, R. 1952. Les Mammiferes pleistocenes de la Republique del Equateur. Memories de la Societe Geologique de France, 66:1-391.

Hoffstetter, R. 1963. La faune Pléistocene de Tarija (Bolivie). Note préliminaire. Bulletin du Musêum National D'Histoire Naturalle, 35:194-203. 
Hoffstetter, R. 1986. High Andean mammalian faunas during the Plio-Pleistocene. In: F. Vuilleumier \& M. Monasterio (eds.) High Altitude Tropical Biogeography, Oxford University Press, p. 218-245.

International Union of Geological Sciences (IUGS) 2012. Request for IUGS Ratification to Establish the Quaternary as a System/ Period of the Cenozoic and revise the associated base of the Pleistocene Series. Available at http://www.iugs.org/

Iriondo, M.H. 1996. Estratigrafía del Cuaternario de la cuenca del río Uruguay. In: CONGRESO GEOLÓGICO ARGENTINO, 13/ CONGRESO DE EXPLORACIÓN DE HIDROCARBUROS, 3, 1996. Actas, Buenos Aires, 4:15-25.

Iriondo, M.H. 2010. Geología del Cuaternario en Argentina. Corrientes, Editorial Moglia, 437 p.

Kerber, L. \& Oliveira, E.V. 2008. Fósseis de vertebrados da Formação Touro Passo (Pleistoceno Superior) Rio Grande do Sul, Brasil: atualização dos dados e novas contribuições. Journal of Geocience, 4:49-64.

Kraglievich, J.L. 1932. Contribución al conocimiento de los ciervos fósiles del Uruguay. Anales del Museo de Historia Natural de Montevideo, 2:355- 438.

Labarca, R.E. \& Alcaraz, M.A. 2011. Presencia de Antifer ultra Ameghino (=Antifer niemeyeri Casamiquela) en el Pleistoceno tardío-Holoceno temprano de Chile central $\left(30-35^{\circ} \mathrm{S}\right)$. Andean Geology, 38:156-170. doi:10.5027/andgeoV38n1-a09

Marshall, L.G.; Berta, A.; Hoffstetter, R.; Pascual, R.; Reig, O. A.; Bombin, M. \& Mones, A. 1984. Mammals and stratigraphy geochronology of the continental mammal-bearing quaternary of South America. Palaeovertebrata, 76 p. (Memoire Extraordinaire).

Martínez, S. \& Ubilla, M. 2004. El Cuaternario de Uruguay. In: G. Veroslavsky; M. Ubilla \& S. Martínez (eds.) Cuencas sedimentarias de Uruguay. Geología, paleontología y recursos naturales. Montevideo, Universidad de la República, Facultad de Ciencias, p. 195-227 (Monografías).

Menegaz, A.N. 2000. Los Camélidos y Cérvidos del Cuaternario del sector Bonaerense de la región Pampeana. Universidad Nacional de La Plata, Argentina, Tesis doctoral, 206 p.

Menegaz, A.N. \& Ortiz-Jaureguizar, E. 1995. Los Artiodáctilos. In: M.T. Alberdi; G. Leone \& E.P. Tonni (eds.) Evolución biológica y climática de la región Pampeana durante los últimos cinco millones de años. Un ensayo de correlación con el Mediterráneo occidental. Madrid, Museo Nacional de Ciencias Naturales. Consejo Superior de Investigaciones Científicas, 311-337 p. (Monografías 12).

Oliveira, E.V. 1992. Mamíferos fósiles do Quaternário do Estado do Rio Grande do Sul, Brasil. Programa de Pos-Graduação em Geociências, Universidade Federal do Rio Grande do Sul, Dissertação de Mestrado, 118 p.

Oliveira, E.V. \& Kerber, L. 2009. Paleontologia e aspectos geológicos das sucessões do final do Neógeno no sudoeste do Rio Grande do Sul, Brasil. Journal of Geocience, 5:21-34.

Paula Couto, C. 1979. Tratado de Paleomastozoología. Rio de Janeiro, Academia Brasileira de Ciências, 590 p.

Ribeiro, A.M. \& Scherer, C.S. 2009. Mamíferos do Pleistoceno do Rio Grande do Sul, Brasil. Ensayo de correlación. In: A.M. Ribeiro; S.G. Bauermann \& C.S. Scherer (orgs.) Quaternário do Rio Grande do Sul. Integrando conhecimentos, p. 171-191 (Monografías da Sociedade Brasileira de Paleontología).

Scillato-Yané, G.J.; Tonni, E.P.; Carlini, A.A. \& Noriega J.I. 1998. Nuevos hallazgos de mamíferos del Cuaternario en el Arroyo Toropí, Corrientes, Argentina. Aspectos Bioestratigráficos, Paleoambientales y Paleozoogeograficos. In: CONGRESO LATINOAMERICANO DE GEOLOGÍA, 6/ CONGRESO NACIONAL DE GEOLOGÍA ECONÓMICA, 10, 1998. Actas, Buenos Aires, 1:263-268.

Scherer, C.S.; Pitana, V.G. \& Ribeiro, A.M. 2007. Novas considerações sobre os Cervidae (Mammalia, Artiodactyla) no Quaternário do Rio Grande do Sul, Brasil. In: JORNADAS ARGENTINAS DE PALEONTOLOGÍA DE VERTEBRADOS, 23, 2007. Libro de Resúmenes, Trelew, p. 32.

Souza Cunha, F.L. \& Magalhães R.M.M. 1981. Cervídeos pleistocénicos de Santa Vitoria do Palmar, Rio Grande do Sul, Brasil. In: CONGRESSO LATINO-AMERICANO PALEONTOLOGÍA, 2, 1981. Anais, Porto Alegre, 2:795-803.

Stehli, F.G. \& Webb, S.D. 1985. The Great American Biotic Interchange. New York, Plenum Press, $532 \mathrm{p}$.

Tomiati, C. \& Abbazzi, L. 2002. Deer fauna from Pleistocene and Holocene localities of Ecuador (South America). Geobios, 35:631-645. doi:10.1016/S0016-6995(02)00075-X

Tonni, E.P; Carlini, A.A; Zurita, A; Frechen, M; Gasparini, G; Budziak, D. \& Kruck, W. 2005. Cronología y bioestratigrafía de las unidades del Pleistoceno aflorantes en el arroyo Toropí, Provincia de Corrientes, Argentina. In: CONGRESSO BRASILEIRO DE PALEONTOLOGÍA, 19/ CONGRESSO LATINO-AMERICANO DE PALEONTOLOGÍA, 6, 2005. Resumen, Aracajú, CD-ROM.

Tonni, E.P. 2009. Las unidades portadoras de vertebrados del Cuaternario de las regiones Mesopotámica y Pampeana Oriental de la Argentina. Ensayo de correlación. In: A.M. Ribeiro; S.G. Bauermann \& C.S. Scherer (orgs.) Quaternário do Rio Grande do Sul. Integrando conhecimentos, p. 57-66 (Monografias da Sociedade Brasileira de Paleontologia).

Ubilla, M. 1996. Paleozoología del Cuaternario continental de la cuenca norte del Uruguay: biogeografía, cronología y aspectos climáticos-ambientales. Universidad de la Republica de Montevideo, Tesis doctoral, $132 \mathrm{p}$.

Ubilla, M. 2004. Mammalian biostratigraphy of Pleistocene fluvial deposits in northern Uruguay, South America. Proceedings of Geologist's Association, 115:347-357. doi:10.1016/S00167878(04)80014-2

Ubilla, M.; Perea, D.; Rinderknecht, A. \& Corona, A. 2009. Pleistocene Mammals from Uruguay: biostratigraphic, biogeographic and environmental connotations. In: A.M. Ribeiro; S.G. Bauermann \& C.S. Scherer (orgs.) Quaternário do Rio Grande do Sul. Integrando conhecimentos, p. 217-230 (Monografias da Sociedade Brasileira de Paleontologia).

Wolf, T.H. 1875. Knochenschlucht von Punin bei Riobamba. Neues Jahrbuch für Mineralogie, Geologie und Paläontologie, p. 1-155.

Zurita, A.E. \& Lutz, A.E. 2002. La Fauna Pleistocena de la Formación Toropí en la Provincia de Corrientes (Argentina). Mastozoología Neotropical, 9:47-56.

Received in June, 2012; accepted in January, 2013. 